\title{
Constraint preserving schemes for some gauge invariant wave equations
}

\author{
Snorre H. Christiansen*
}

May 8, 2007

\begin{abstract}
We study discretizations of the Maxwell-Klein-Gordon equation as an example of a constrained geometric non-linear evolution partial differential equation. For the temporal gauge we propose a fully discrete scheme which preserves the non-linear constraint thanks to a special application of Lagrange multipliers. We show that the method generalizes to Hamiltonian wave equations whose kinetic and potential energy are both invariant under a group of transformations, even though the Galerkin spaces are not invariant. We then extend the method to the Lorenz gauge. Numerical results illustrate the discussion.
\end{abstract}

\section{Introduction}

Many physical phenomena can be modeled by an evolution equation for a constrained field. In particular the vacuum Maxwell equation and the incompressible Navier-Stokes equation are evolution equations for divergence-free vector fields. For both these equations the discretizations in terms of mixed finite elements - and other methods - is relatively well understood (see [8][12][14][23]). In other cases the situation is less favorable. In particular current simulations of the Einstein equation for general relativity are less successful. One reason is that, contrary to the two preceding examples, the constraint is non-linear. More generally, problems seem to arise when the equation describes the geometry itself rather than an object living on it. The way the geometry interacts with itself leads to non-linear equations.

In order to gain insight in the problem of constraint preservation for nonlinear equations we studied the Yang-Mills (YM) equation in [9]. It is a geometric equation in the sense that it describes a connection, providing the covariant derivative other fields are subject to. For non-commutative gauge groups, such as $\mathrm{SU}(2)$, the connection satisfies a non-linear evolution equation because the curvature contains a quadratic term. The Gauss law and the Bianchi identity are both non-linear constraints the connection should satisfy. In spite of these difficulties we were able to design constraint-preserving discretizations. However the construction appeared to use a special cancellation property of the bracket

\footnotetext{
${ }^{*}$ CMA, University of Oslo, PO Box 1053 Blindern, NO-0316 Oslo, Norway. email :
} snorrec@math .uio.no 
of Lie algebra valued differential forms. This seemed to limit the scope of the article.

The simplest extension of the Yang-Mills equation consists in adding a vector valued so-called Higgs field, subject to a wave equation with covariant derivatives given by the connection. In the special case where the gauge group is $\mathrm{U}(1)$, the Yang-Mills-Higgs equation reduces to the Maxwell-Klein-Gordon (MKG) equation. Even though the Maxwell equation is linear and has linear constraints, this is a non-linear evolution equation with a nonlinear constraint generalizing the Gauss law. It is also geometric in nature. The Bianchi identity, expressing the absence of magnetic monopoles, is linear however. This article is an investigation of the discretization of the MKG equation. It is a sequel to [9], continuing our investigation of discretizations of constrained geometric non-linear evolution partial differential equations.

We first investigate the MKG equation in temporal gauge. We introduce discretizations based on mixed finite elements, or, equivalently, Whitney forms. It appears that simply restricting the variational principle to such spaces produces a constraint-violating scheme, even without time-discretization, as we already observed for the Yang-Mills equation. That is, simple space-discretization conflicts with time-conservation of the constraint. However we are able to correct for this by introducing Lagrange multipliers in a special, gentle, way. We achieve constraint preservation both for the semi-discrete scheme and the timediscretized one. The method is similar to the one we derived for the Yang-Mills equation, but the construction involves another type of cancellation.

The constraint-preserving nature of the evolution equation can be related, via Noether's theorem, to the invariance of the Lagrangian under gauge transformations. The constraint-violating nature of simple variational discretizations can then be explained by the non-invariance of the Galerkin spaces under the natural subgroup of gauge transformations. In this paper we carry this line of investigation further and explain the good behavior of the Lagrange multiplier constrained scheme, by the properties of the group action. We show that for any Lagrangian whose kinetic and potential terms are separately invariant under a group of transformations, we can produce a fully discrete constraint-preserving scheme, even though the Galerkin spaces are not invariant under the group action. In particular we obtain a unified interpretation of the above mentioned cancellation properties for the YM and MKG equations.

Then we turn our attention to non-zero gauges. We first argue that in order to have a good representation of the conjugate momentum of the complex scalar field one should perform what is called a mass-lumping. We then introduce space-discretizations. They turn out not to be energy preserving. We nevertheless extend the constraint method to this more general gauge and perform some numerical experiments in the Lorenz gauge. First, without Lagrange multipliers it appears that the non-conservation of energy is not catastrophic but that, as for the temporal gauge, there is a substantial drift in the total charge - in other words the constraint is violated. With Lagrange multipliers the energy is almost identical but the constraint is now satisfied (though we could prove this only for the space semi-discretization).

The article is organized as follows: In $\S 2$ we introduce the Maxwell-KleinGordon equation and prove energy and constraint preservation. In $\S 3$ we discuss discretizations in the temporal gauge. We compare unconstrained and constrained formulations theoretically and numerically. We generalize our findings 
concerning the MKG equation to certain gauge invariant Hamiltonian systems. In $\S 4$ we turn to non-zero gauges and discuss also unconstrained and constrained formulations.

\section{Maxwell-Klein-Gordon equation}

Let $M$ be a compact Riemannian manifold which for simplicity we take to have no boundary. Alternatively one can think of $M$ as a bounded domain in $\mathbb{R}^{3}$ with a Lipshitz boundary and incorporate Dirichlet boundary conditions where appropriate. The real valued $\mathrm{L}^{2}$ scalar product on differential forms on $M$ is denoted $\langle\cdot, \cdot\rangle$, and the associated $\mathrm{L}^{2}$ norm is denoted $|\cdot|$. All adjoints will be taken with respect to these $\mathrm{L}^{2}$ products.

The unknowns of the theory are on the one side a time-dependent gauge potential $t \mapsto \alpha(t) \mathrm{d} t+A(t)$ such that $\alpha(t)$ is a purely imaginary function on $M$ (an element of $\Omega^{0}(M) \otimes i \mathbb{R}$ ) and $A(t)$ is a purely imaginary one-form on $M$ (an element of $\left.\Omega^{1}(M) \otimes i \mathbb{R}\right)$, and on the other side a time-dependent complex scalar function $t \mapsto \phi(t)$ on $M$ (an element of $\Omega^{0}(M) \otimes \mathbb{C}$ ). The action is given by:

$$
\mathcal{S}(A, \phi, \alpha)=(1 / 2) \int-|\mathrm{d} \alpha-\dot{A}|^{2}+|\mathrm{d} A|^{2}-|\dot{\phi}+\alpha \phi|^{2}+|\mathrm{D} \phi+A \phi|^{2} .
$$

Here $\mathrm{d}: \Omega^{k} \otimes i \mathbb{R} \rightarrow \Omega^{k+1} \otimes i \mathbb{R}$ denotes the exterior derivative on purely imaginary differential forms and $\mathrm{D}: \Omega^{0} \otimes \mathbb{C} \rightarrow \Omega^{1} \otimes \mathbb{C}$ is the (full) differential of complex scalar fields.

The variation of $\mathcal{S}$ at $(A, \phi, \alpha)$ in direction $\left(A^{\prime}, \phi^{\prime}, \alpha^{\prime}\right)$ is given by :

$$
\begin{aligned}
& \mathrm{D} \mathcal{S}(A, \phi, \alpha)\left(A^{\prime}, \phi^{\prime}, \alpha^{\prime}\right) \\
= & \int-\left\langle\mathrm{d} \alpha-\dot{A}, \mathrm{~d} \alpha^{\prime}-\dot{A}^{\prime}\right\rangle+\left\langle\mathrm{d} A, \mathrm{~d} A^{\prime}\right\rangle+ \\
& \int-\left\langle\dot{\phi}+\alpha \phi, \dot{\phi}^{\prime}+\alpha \phi^{\prime}+\alpha^{\prime} \phi\right\rangle+\left\langle\mathrm{D} \phi+A \phi, \mathrm{D} \phi^{\prime}+A \phi^{\prime}+A^{\prime} \phi\right\rangle .
\end{aligned}
$$

We introduce the covariant derivative on complex fields on $M$ associated with the gauge potential $A$ :

$$
\mathrm{D}_{A}: \phi \mapsto \mathrm{D} \phi+A \phi
$$

We also introduce the purely imaginary part operator on $\mathbb{C}$ :

$$
\mathfrak{I}: a+b i \mapsto b i, \quad \text { for } \quad a, b \in \mathbb{R} .
$$

Fields $E$ and $\psi$ are defined by:

$$
E(t)=-\dot{A}(t)+\mathrm{d} \alpha(t),
$$

and:

$$
\psi(t)=-\dot{\phi}(t)-\alpha(t) \phi(t) .
$$

These fields are the momenta conjugate to $A(t)$ and $\phi(t)$ respectively.

Stationarity of the action provides the following Euler-Lagrange equations: Variations with respect to $A^{\prime}$ provide the evolution equation:

$$
\dot{E}(t)=\mathrm{d}^{\star} \mathrm{d} A(t)+\Im\left(\mathrm{D}_{A(t)} \phi(t) \bar{\phi}(t)\right) .
$$


Variations with respect to $\phi^{\prime}$ provide, after integration by parts:

$$
\dot{\psi}(t)+\alpha(t) \psi(t)=\mathrm{D}_{A(t)}^{\star} \mathrm{D}_{A(t)} \phi(t) .
$$

On the other hand variations with respect to $\alpha^{\prime}$ provide the constraint:

$$
\mathrm{d}^{\star} E(t)-\mathfrak{I}(\psi(t) \bar{\phi}(t))=0 .
$$

No evolution equation for $t \mapsto \alpha(t)$ is provided. It may be regarded as an essentially arbitrary function. In the following propositions we suppose that $\alpha$ is given.

An important property we will use many times is that for any fields $\Phi, \Psi$ and any purely imaginary $\alpha$ we have:

$$
\langle\alpha \Phi, \Psi\rangle+\langle\Phi, \alpha \Psi\rangle=0,
$$

in particular for any field $\Phi$ and any purely imaginary $\alpha$ :

$$
\langle\alpha \Phi, \Phi\rangle=0 .
$$

Proposition 2.1 Suppose $(A, \phi, E, \psi)$ solves the evolution equations $(5,6,7$, 8) on a time-interval $[0, T]$. Suppose furthermore that the constraint (9) is satisfied at time $t=0$. Then the constraint (9) is satisfied for all $t \in[0, T]$.

- Proof: We write, for any purely imaginary function $\alpha^{\prime}$ which is constant in time:

$$
\begin{aligned}
& \left(\left\langle E, \mathrm{~d} \alpha^{\prime}\right\rangle-\left\langle\psi \bar{\phi}, \alpha^{\prime}\right\rangle\right)^{.} \\
= & \left\langle\dot{E}, \mathrm{~d} \alpha^{\prime}\right\rangle-\left\langle\dot{\psi} \bar{\phi}+\psi \dot{\bar{\phi}}, \alpha^{\prime}\right\rangle, \\
= & \left\langle\mathrm{D}_{A} \phi \bar{\phi}, \mathrm{d} \alpha^{\prime}\right\rangle-\left\langle\left(\mathrm{D}_{A}^{\star} \mathrm{D}_{A} \phi-\alpha \psi\right) \bar{\phi}, \alpha^{\prime}\right\rangle+\left\langle\psi(\overline{\alpha \phi+\psi}), \alpha^{\prime}\right\rangle, \\
= & \left\langle\mathrm{D}_{A} \phi, \phi \mathrm{d} \alpha^{\prime}\right\rangle-\left\langle\mathrm{D}_{A} \phi, \mathrm{D}_{A}\left(\phi \alpha^{\prime}\right)\right\rangle+\left\langle\alpha \psi \bar{\phi}, \alpha^{\prime}\right\rangle-\left\langle\alpha \psi \bar{\phi}, \alpha^{\prime}\right\rangle, \\
= & \left\langle\mathrm{D}_{A} \phi, \phi \mathrm{d} \alpha^{\prime}\right\rangle-\left\langle\mathrm{D}_{A} \phi,\left(\mathrm{D}_{A} \phi\right) \alpha^{\prime}+\phi \mathrm{d} \alpha^{\prime}\right\rangle, \\
= & 0 .
\end{aligned}
$$

This concludes the proof.

The energy of the system at any time $t$ is:

$$
\mathcal{H}(A, \phi, E, \psi)=(1 / 2)\left(\langle E, E\rangle+\langle\mathrm{d} A, \mathrm{~d} A\rangle+\langle\psi, \psi\rangle+\left\langle\mathrm{D}_{A} \phi, \mathrm{D}_{A} \phi\right\rangle\right) .
$$

Proposition 2.2 Suppose $(A, \phi, E, \psi)$ solves the evolution equations (5, 6, 7, 8). Then energy is conserved.

- Proof: We write:

$$
\begin{aligned}
\dot{\mathcal{H}}= & \langle E, \dot{E}\rangle+\langle\mathrm{d} A, \mathrm{~d} \dot{A}\rangle+\langle\psi, \dot{\psi}\rangle+\left\langle\mathrm{D}_{A} \phi, \mathrm{D}_{A} \dot{\phi}\right\rangle+\left\langle\mathrm{D}_{A} \phi, \dot{A} \phi\right\rangle, \\
= & \langle\mathrm{d} E, \mathrm{~d} A\rangle+\left\langle E \phi, \mathrm{D}_{A} \phi\right\rangle-\langle\mathrm{d} A, \mathrm{~d} E\rangle+\left\langle\mathrm{D}_{A} \psi, \mathrm{D}_{A} \phi\right\rangle \\
& +\left\langle\mathrm{D}_{A} \phi, \mathrm{D}_{A}(-\psi-\alpha \phi)\right\rangle+\left\langle\mathrm{D}_{A} \phi,(-E+\mathrm{d} \alpha) \phi\right\rangle, \\
= & -\left\langle\mathrm{D}_{A} \phi, \alpha \mathrm{D}_{A} \phi\right\rangle-\left\langle\mathrm{D}_{A} \phi, \mathrm{d} \alpha \phi\right\rangle+\left\langle\mathrm{D}_{A} \phi, \mathrm{d} \alpha \phi\right\rangle, \\
= & 0 .
\end{aligned}
$$

This concludes the proof. 


\section{Discretization in temporal gauge}

\subsection{Variational discretization}

We investigate first the temporal gauge, which is to impose $\alpha(t)=0$ at all times $t$. In this gauge the equations we obtain are:

$$
\begin{aligned}
\dot{A} & =-E, \\
\dot{\phi} & =-\psi, \\
\dot{E} & =\mathrm{d}^{\star} \mathrm{d} A+\Im\left(\mathrm{D}_{A} \phi \bar{\phi}\right), \\
\dot{\psi} & =\mathrm{D}_{A}^{\star} \mathrm{D}_{A} \phi .
\end{aligned}
$$

These equations can be seen to be associated with the Lagrangian:

$$
\mathcal{L}(A, \phi, \dot{A}, \dot{\phi})=(1 / 2)\left(-|\dot{A}|^{2}+|\mathrm{d} A|^{2}-|\dot{\phi}|^{2}+\left|\mathrm{D}_{A} \phi\right|^{2}\right) .
$$

We first perform space-discretization. We consider semi-discretizations of (24 - 27) based on finite dimensional spaces $X_{\sigma}=Y_{\sigma}^{1} \times Z_{\sigma}^{0}$ such that $Y_{\sigma}^{1}$ is a space of gauge potentials and $Z_{\sigma}^{0}$ is a space of complex functions. In the numerical experiments we suppose that $M$ is equipped with a simplicial mesh. We let $Z_{\sigma}^{0}$ be the space of complex piece-wise affine and continuous scalar functions (it has one complex degree of freedom per vertex). We also let $Y_{\sigma}^{1}$ be the space of (purely imaginary) Whitney one-forms on $M$ (it has one real degree of freedom per edge). For material on $Y_{\sigma}^{1}$ we refer to [26][24][22][6] and more recent surveys in $[14][21]$.

It is natural to look for functions $t \mapsto(A(t), \phi(t)) \in X_{\sigma}$ such that on any time-interval the action is critical at this function among variations that preserve the end-points and take values in $X_{\sigma}$. This leads to the following discrete EulerLagrange equations: for all $E^{\prime} \in Y_{\sigma}^{1}$, all $\psi^{\prime} \in Z_{\sigma}^{0}$ :

$$
\begin{aligned}
\dot{A} & =-E, \\
\dot{\phi} & =-\psi, \\
\left\langle\dot{E}, E^{\prime}\right\rangle & =\left\langle\mathrm{d} A, \mathrm{~d} E^{\prime}\right\rangle+\left\langle\mathrm{D}_{A} \phi, \phi E^{\prime}\right\rangle \\
\left\langle\dot{\psi}, \psi^{\prime}\right\rangle & =\left\langle\mathrm{D}_{A} \phi, \mathrm{D}_{A} \psi^{\prime}\right\rangle .
\end{aligned}
$$

Do these equations preserve the constraint (9) in some form? For standard finite-element spaces such as the ones we consider, $\mathrm{d}^{\star}$ maps $Y_{\sigma}^{1}$ into a space of non-integrable measures hinting that one should try to conserve moments of the charge. There is a space $Y_{\sigma}^{0}$ such that $\mathrm{d}: Y_{\sigma}^{0} \rightarrow Y_{\sigma}^{1}$ and $Y_{\sigma}^{0}$ is maximal with this property. For our choice of $Y_{\sigma}^{1}, Y_{s}^{0}$ igma is simply the space of purely imaginary piecewise affine and continuous functions (it has one real degree of freedom per vertex). Now one can ask if for each $\alpha^{\prime} \in Y_{\sigma}^{0}$ the quantities:

$$
\left\langle E, \mathrm{~d} \alpha^{\prime}\right\rangle-\left\langle\psi, \phi \alpha^{\prime}\right\rangle,
$$

are conserved. We remark that the proof of Proposition 2.1 relies essentially on the possibility, given $\alpha^{\prime}$, to insert $E^{\prime}=\mathrm{d} \alpha^{\prime}$ in (31) and $\psi^{\prime}=-\phi \alpha^{\prime}$ in (32), and add the two equations. But contrary to the continuous case this is not legitimate, since in general $\phi \alpha^{\prime}$ is not in $Z_{\sigma}^{0}$. This kind of problem is similar to the one encountered for the Yang-Mills equations. Before continuing we 
check numerically if this illegitimacy leads to problems, that is, if charge is approximately conserved or not.

We now perform a time-discretization. The most popular method of discretizing Hamiltonian systems is by the leap-frog scheme; see [13][19] for the case of ordinary differential equations and [15] for applications to wave equations. In our case this consists in choosing a (fixed) time-step $\tau$ and computing, for integer $n$, approximations $A_{n} \simeq A(n \tau)$ and $\phi_{n} \simeq \phi(n \tau)$ as well as $E_{n+1 / 2} \simeq E((n+1 / 2) \tau)$ and $\psi_{n+1 / 2} \simeq \psi((n+1 / 2) \tau)$ by the induction formulas:

$$
\begin{aligned}
(1 / \tau)\left(A_{n}-A_{n-1}\right) & =-E_{n-1 / 2}, \\
(1 / \tau)\left(\phi_{n}-\phi_{n-1}\right) & =-\psi_{n-1 / 2}, \\
(1 / \tau)\left(E_{n+1 / 2}-E_{n-1 / 2}\right) & =\dot{E}_{n}, \\
(1 / \tau)\left(\psi_{n+1 / 2}-\psi_{n-1 / 2}\right) & =\dot{\psi}_{n},
\end{aligned}
$$

where $\dot{E}_{n} \in Y_{\sigma}$ and $\dot{\psi}_{n} \in Z_{\sigma}^{0}$ are solutions of, for all $E^{\prime} \in Y_{\sigma}$ :

$$
\left\langle\dot{E}_{n}, E^{\prime}\right\rangle=\left\langle\mathrm{d} A_{n}, \mathrm{~d} E^{\prime}\right\rangle+\left\langle\mathrm{D}_{A_{n}} \phi_{n}, \phi_{n} E^{\prime}\right\rangle,
$$

and all $\psi^{\prime} \in Z_{\sigma}^{0}$ :

$$
\left\langle\dot{\psi}_{n}, \psi^{\prime}\right\rangle=\left\langle\mathrm{D}_{A_{n}} \phi_{n}, \mathrm{D}_{A_{n}} \psi^{\prime}\right\rangle .
$$

For the numerical experiments we have chosen $M$ to be the two-dimensional sphere $S^{2}$ equipped with the metric inherited from $\mathbb{R}^{3}$. In all figures we plot functions of time in multiples of $\pi$. Notice that a linear wave with propagation speed one travels around the sphere in two time-units.

In Figure 1 we plot the energy of the solution. At time-step $n$ it is defined to by:

$$
2 \mathcal{H}_{n}=\left|\frac{E_{n+1 / 2}+E_{n-1 / 2}}{2}\right|^{2}+\left|\mathrm{d} A_{n}\right|^{2}+\left|\frac{\psi_{n+1 / 2}+\psi_{n-1 / 2}}{2}\right|^{2}+\left|\mathrm{D}_{A_{n}} \phi_{n}\right|^{2} .
$$

As is well-known in the study of Hamiltonian ODEs, the energy is not exactly conserved but it is nearly conserved over long times.

Next, in Figure 2, we plot the $\mathrm{L}^{2}$ norm squared of the constraint field $\gamma_{n}$, defined by:

$$
\left\langle\gamma_{n}, \beta^{\prime}\right\rangle=\left\langle\frac{E_{n+1 / 2}+E_{n-1 / 2}}{2}, \mathrm{~d} \beta^{\prime}\right\rangle-\left\langle\frac{\psi_{n+1 / 2}+\psi_{n-1 / 2}}{2}, \phi_{n} \beta^{\prime}\right\rangle
$$

We compare it with the divergence of the electric field $\delta_{n}$, defined by:

$$
\left\langle\delta_{n}, \beta^{\prime}\right\rangle=\left\langle\frac{E_{n+1 / 2}+E_{n-1 / 2}}{2}, \mathrm{~d} \beta^{\prime}\right\rangle .
$$

While ideally $\gamma_{n}$ should be $0, \delta_{n}$ should not. As we can see the constraint is not satisfied and rather quickly the constraint field $\gamma_{n}$ attains a magnitude comparable to that of $\delta_{n}$. In other words the noise becomes comparable to the signal.

\subsection{Gentle constraints}

As in the case of Yang-Mills equations we correct for the non-satisfaction of the constraint by enforcing it in incremental form. That is, we enforce the constraint 


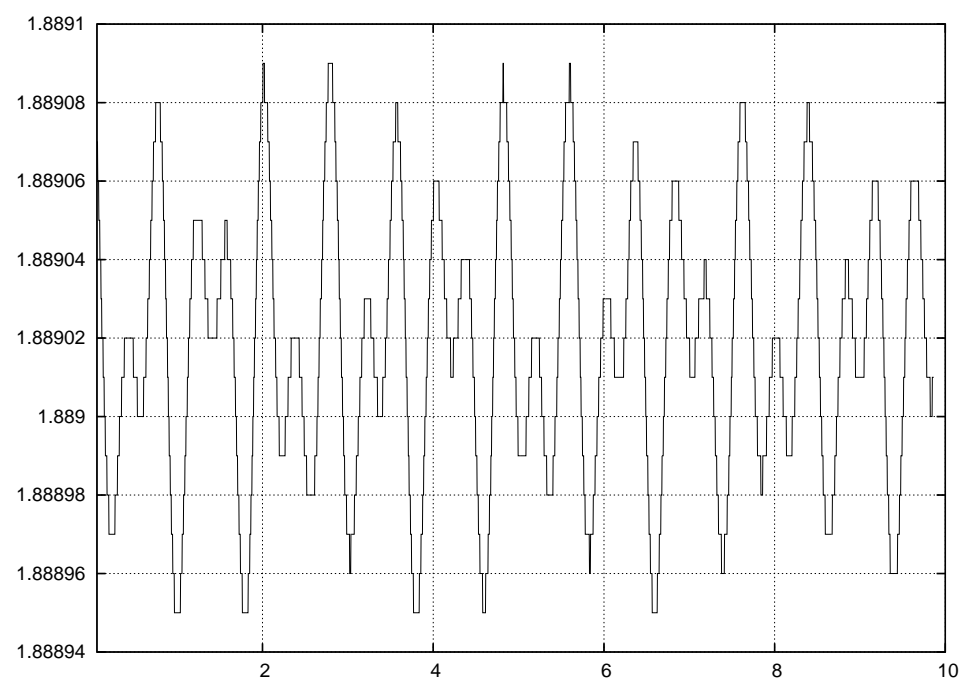

Figure 1: Energy; unconstrained, temporal gauge.

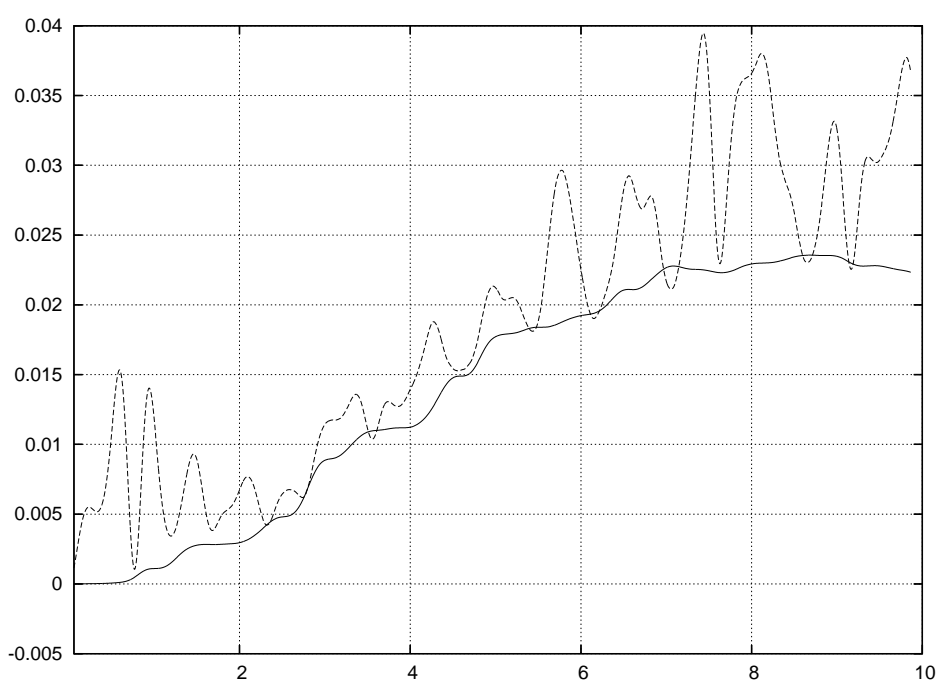

Figure 2: Constraint (plain) and divergence (dashed); unconstrained, temporal gauge. 
to be constant, so that if it's satisfied initially it will be satisfied at later times. For this purpose we use Lagrange multipliers so that testing with $E^{\prime}=\mathrm{d} \beta^{\prime}$ and $\psi^{\prime}=\phi \beta^{\prime}$ (which we recall was illegitimate in $(31-31)$ ) gives the desired result, namely 0 . In other words we enforce:

$$
\left\langle\dot{E}, \mathrm{~d} \beta^{\prime}\right\rangle-\left\langle\dot{\psi}, \phi \beta^{\prime}\right\rangle-\left\langle\psi, \dot{\phi} \beta^{\prime}\right\rangle=0,
$$

taking into account that we should have:

$$
\left\langle\psi, \dot{\phi} \beta^{\prime}\right\rangle=-\left\langle\psi, \psi \beta^{\prime}\right\rangle=0
$$

We keep the first part of the equation:

$$
\begin{aligned}
\dot{A} & =-E, \\
\dot{\phi} & =-\psi,
\end{aligned}
$$

The two last equations are replaced by the mixed problem, find $\dot{E} \in Y_{\sigma}^{1}, \dot{\psi} \in Z_{\sigma}^{0}$ and a Lagrange multiplier $\beta \in Y_{\sigma}^{0}$ such that for all $E^{\prime} \in Y_{\sigma}^{1}$, all $\psi^{\prime} \in Z_{\sigma}^{0}$ and all $\beta^{\prime} \in Y_{h}^{0}$ :

$$
\begin{array}{ll}
\left\langle\dot{E}, E^{\prime}\right\rangle+\left\langle\dot{\psi}, \psi^{\prime}\right\rangle+\left\langle E^{\prime}, \mathrm{d} \beta\right\rangle-\left\langle\psi^{\prime}, \phi \beta\right\rangle & =f_{t}\left(E^{\prime}\right)+g_{t}\left(\psi^{\prime}\right), \\
\left\langle\dot{E}, \mathrm{~d} \beta^{\prime}\right\rangle-\left\langle\dot{\psi}, \phi \beta^{\prime}\right\rangle & =0 .
\end{array}
$$

with:

$$
f_{t}\left(E^{\prime}\right)=\left\langle\mathrm{d} A, \mathrm{~d} E^{\prime}\right\rangle+\left\langle\mathrm{D}_{A} \phi, \phi E^{\prime}\right\rangle,
$$

and:

$$
g_{t}\left(\psi^{\prime}\right)=\left\langle\mathrm{D}_{A} \phi, \mathrm{D}_{A} \psi^{\prime}\right\rangle
$$

We first remark that since $\mathrm{d}$ is a map $Y_{\sigma}^{0} \rightarrow Y_{\sigma}^{1}$ we have:

Proposition 3.1 The saddle-point problem (47) satisfies a Babuska-Brezzi compatibility condition:

$$
\inf _{\beta^{\prime} \in Y_{\sigma}^{0}} \sup _{E^{\prime} \in Y_{\sigma}^{1}, \psi^{\prime} \in Z_{\sigma}^{0}} \frac{\left\langle E^{\prime}, \mathrm{d} \beta^{\prime}\right\rangle-\left\langle\psi^{\prime}, \phi \beta^{\prime}\right\rangle}{\left(\left|\beta^{\prime}\right|^{2}+\left|\mathrm{d} \beta^{\prime}\right|^{2}\right)^{1 / 2}\left(\left|E^{\prime}\right|^{2}+\left|\psi^{\prime}\right|^{2}\right)^{1 / 2}} \geq 1 / C .
$$

More interestingly:

Proposition 3.2 Equations (45, 46, 47) preserve the constraint:

$$
\left\langle E, \mathrm{~d} \beta^{\prime}\right\rangle-\left\langle\psi, \phi \beta^{\prime}\right\rangle=0
$$

as well as the Hamiltonian.

- Proof: Taking into account the constraint on $(\dot{E}, \dot{\psi})$ :

$$
\begin{aligned}
\left(\left\langle E, \mathrm{~d} \beta^{\prime}\right\rangle-\left\langle\psi, \phi \beta^{\prime}\right\rangle\right)^{\cdot} & =-\left\langle\psi, \dot{\phi} \beta^{\prime}\right\rangle, \\
& =\left\langle\psi, \psi \beta^{\prime}\right\rangle, \\
& =0 .
\end{aligned}
$$

This proves constraint preservation. 
To prove that the Hamiltonian is preserved one now puts $E^{\prime}=E$ and $\psi^{\prime}=\psi$ in (47), and remarks that the term with the Lagrange multiplier vanishes since the constraint is satisfied. That is:

$$
\begin{aligned}
& \mathcal{H}(A, \phi, E, \psi)^{\circ} \\
= & \langle E, \dot{E}\rangle+\langle\mathrm{d} A, \mathrm{~d} \dot{A}\rangle+\langle\psi, \dot{\psi}\rangle+\left\langle\mathrm{D}_{A} \phi,\left(\mathrm{D}_{A} \phi\right)^{\circ}\right\rangle, \\
= & \langle E, \dot{E}\rangle+\langle\psi, \dot{\psi}\rangle-\langle\mathrm{d} A, \mathrm{~d} E\rangle-\left\langle\mathrm{D}_{A} \phi, \mathrm{D}_{A} \psi\right\rangle-\left\langle\mathrm{D}_{A} \phi, E \phi\right\rangle, \\
= & 0 .
\end{aligned}
$$

This concludes the proof.

Turning to time-discretizations we consider the following modification of the leap-frog scheme. We keep:

$$
\begin{aligned}
(1 / \tau)\left(A_{n}-A_{n-1}\right) & =-E_{n-1 / 2}, \\
(1 / \tau)\left(\phi_{n}-\phi_{n-1}\right) & =-\psi_{n-1 / 2}, \\
(1 / \tau)\left(E_{n+1 / 2}-E_{n-1 / 2}\right) & =\dot{E}_{n}, \\
(1 / \tau)\left(\psi_{n+1 / 2}-\psi_{n-1 / 2}\right) & =\dot{\psi}_{n},
\end{aligned}
$$

but now $\dot{E}_{n} \in Y_{\sigma}$ and $\dot{\psi}_{n} \in Z_{\sigma}^{0}$ are solutions of, for a certain Lagrange multiplier $\beta_{n} \in Y_{\sigma}^{0}$, for all $E^{\prime} \in Y_{\sigma}^{1}$, all $\psi^{\prime} \in Z_{\sigma}^{0}$ and all $\beta^{\prime} \in Y_{\sigma}^{0}$ :

$$
\begin{array}{ll}
\left\langle\dot{E}_{n}, E^{\prime}\right\rangle+\left\langle\dot{\psi}_{n}, \psi^{\prime}\right\rangle+\left\langle E^{\prime}, \mathrm{d} \beta_{n}\right\rangle-\left\langle\psi^{\prime}, \phi_{n} \beta_{n}\right\rangle & =f_{n}\left(E^{\prime}\right)+g_{n}\left(\psi^{\prime}\right), \\
\left\langle\dot{E}_{n}, \mathrm{~d} \beta^{\prime}\right\rangle-\left\langle\dot{\psi}_{n}, \phi_{n} \beta^{\prime}\right\rangle & =0,
\end{array}
$$

with:

$$
\begin{aligned}
f_{n}\left(E^{\prime}\right) & =\left\langle\mathrm{d} A_{n}, \mathrm{~d} E^{\prime}\right\rangle+\left\langle\mathrm{D}_{A_{n}} \phi_{n}, \phi_{n} E^{\prime}\right\rangle, \\
g_{n}\left(\psi^{\prime}\right) & =\left\langle\mathrm{D}_{A_{n}} \phi_{n}, \mathrm{D}_{A_{n}} \psi^{\prime}\right\rangle .
\end{aligned}
$$

Remark first that by the constraint on $\left(\dot{E}_{n}, \dot{\psi}_{n}\right)$ we have:

$$
\begin{aligned}
& \left\langle E_{n+1 / 2}, \mathrm{~d} \beta^{\prime}\right\rangle-\left\langle\psi_{n+1 / 2}, \phi_{n} \beta^{\prime}\right\rangle \\
= & \left\langle\frac{E_{n+1 / 2}+E_{n-1 / 2}}{2}, \mathrm{~d} \beta^{\prime}\right\rangle-\left\langle\frac{\psi_{n+1 / 2}+\psi_{n-1 / 2}}{2}, \phi_{n} \beta^{\prime}\right\rangle .
\end{aligned}
$$

We have:

Proposition 3.3 For each $\beta^{\prime} \in Y_{\sigma}^{0}$ the constrained leap-frog scheme (59-62, 63) preserves the quantity:

$$
\left\langle E_{n+1 / 2}, \mathrm{~d} \beta^{\prime}\right\rangle-\left\langle\psi_{n+1 / 2}, \phi_{n} \beta^{\prime}\right\rangle .
$$

- Proof: Indeed we have:

$$
\begin{aligned}
& \left\langle E_{n+1 / 2}, \mathrm{~d} \beta^{\prime}\right\rangle-\left\langle\psi_{n+1 / 2}, \phi_{n} \beta^{\prime}\right\rangle \\
= & \left\langle E_{n-1 / 2}, \mathrm{~d} \beta^{\prime}\right\rangle-\left\langle\psi_{n-1 / 2}, \phi_{n} \beta^{\prime}\right\rangle, \\
= & \left\langle E_{n-1 / 2}, \mathrm{~d} \beta^{\prime}\right\rangle-\left\langle\psi_{n-1 / 2},\left(\phi_{n-1}-\tau \psi_{n-1 / 2}\right) \beta^{\prime}\right\rangle, \\
= & \left\langle E_{n-1 / 2}, \mathrm{~d} \beta^{\prime}\right\rangle-\left\langle\psi_{n-1 / 2}, \phi_{n-1} \beta^{\prime}\right\rangle .
\end{aligned}
$$

This concludes the proof.

In Figure 3 plot the energy of the solution. The similarity with the unconstrained energy plot is striking.

Next, in Figure 4 we plot the $\mathrm{L}^{2}$ norm squared of the constraint field, as well as that the divergence of the electric field. As predicted the constraint is now satisfied. 


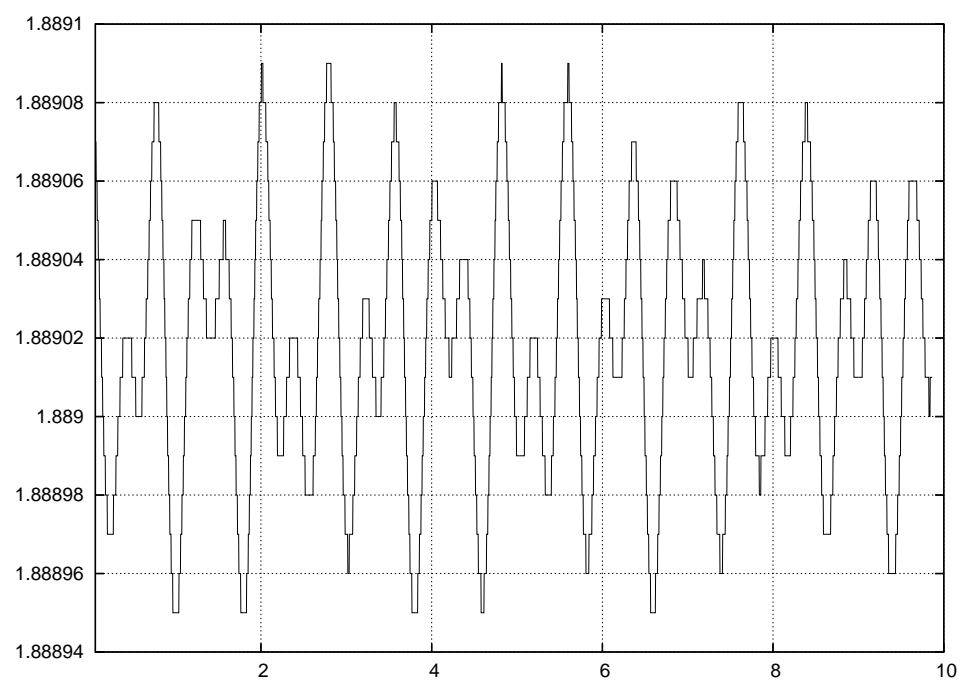

Figure 3: Energy; constrained, temporal gauge.

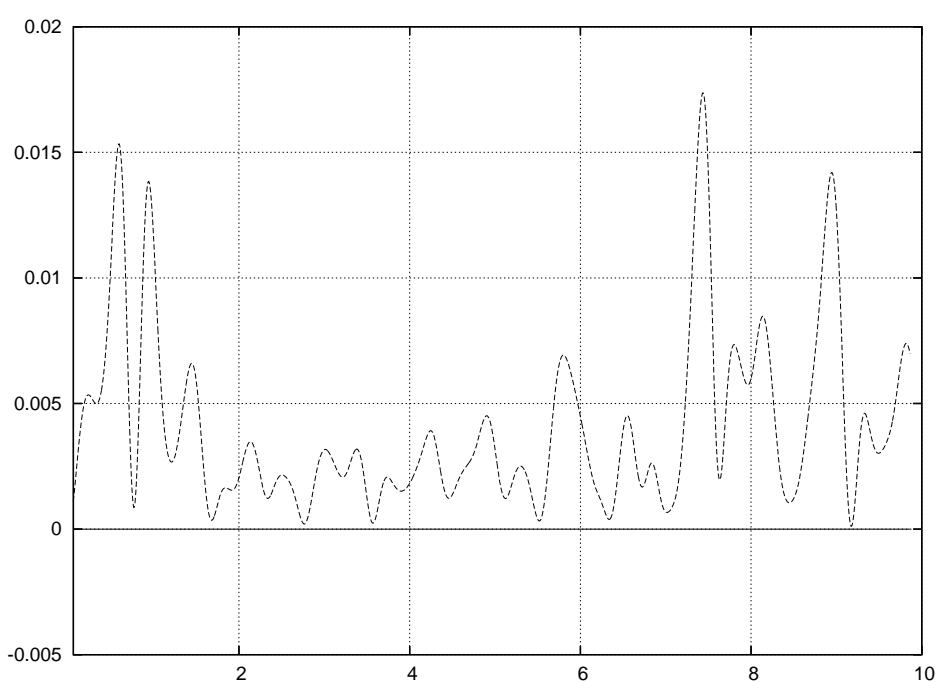

Figure 4: Constraint (plain) and divergence (dashed); constrained, temporal gauge. 


\subsection{Gauge transformations}

In [9] we related the preservation of the constraint of the continuous Yang-Mills equation to the invariance of the Lagrangian under gauge transformations. A similar interpretation is possible here. For each purely imaginary function $\beta$, each gauge potential $A$ and each scalar field $\phi$, we put:

$$
\mathcal{G}_{\beta}(A, \phi)=(A-\mathrm{d} \beta,(\exp \beta) \phi)
$$

One checks that $\mathcal{G}$ is a group action:

$$
\mathcal{G}_{\beta+\beta^{\prime}}(A, \phi)=\mathcal{G}_{\beta}\left(\mathcal{G}_{\beta^{\prime}}(A, \phi)\right)
$$

Moreover we have:

$$
\mathrm{D}_{A-\mathrm{d} \beta}((\exp \beta) \phi)=(\exp \beta) \mathrm{D}_{A} \phi .
$$

From this it follows that:

$$
\mathcal{L}\left(\mathcal{G}(A, \phi), \mathrm{DG}_{\beta}(A, \phi)\left(A^{\prime}, \phi^{\prime}\right)\right)=\mathcal{L}\left(A, \phi, A^{\prime}, \phi^{\prime}\right) .
$$

Now, in the continuous case, this invariance property entails the constraint preservation by Noether's theorem. In the discrete case however, Noether's theorem does not apply because, for $\beta \in Y_{\sigma}^{0}, \mathcal{G}_{\beta}$ does not map $X_{\sigma}$ to itself. However it is possible to provide an interpretation , in terms of the group action, of the gentle constraint method we just introduced.

The infinitesimal generators of $\mathcal{G}$ are the $\Gamma(A, \phi)$ defined by:

$$
\Gamma(A, \phi) \beta^{\prime}=\left.\partial_{\beta^{\prime}} \mathcal{G}_{\beta}(A, \phi)\right|_{\beta=0} .
$$

Explicitly we have:

$$
\Gamma(A, \phi) \beta^{\prime}=\left(-\mathrm{d} \beta^{\prime}, \beta^{\prime} \phi\right) .
$$

This expression appears in the constraint of our saddle-point formulation; it has the form:

$$
\left\langle(\dot{E}, \dot{\psi}), \Gamma(A, \phi) \beta^{\prime}\right\rangle=0 .
$$

We also remark that for $(A, \phi) \in X_{\sigma}$ and $\beta^{\prime} \in Y_{\sigma}^{0}$ the vector $\Gamma(A, \phi) \beta^{\prime}$ points out of $X_{\sigma}$ but not orthogonally, by the Babuska-Brezzi compatibility condition. In the next section we discuss this situation in a more general setting.

\subsection{Generalization to invariant Lagrangians}

Suppose we have a configuration space $X$ which is a vector space equipped with a positive definite symmetric bilinear form $\langle\cdot, \cdot\rangle$ and a Lagrangian $\mathcal{L}: X \times X \rightarrow \mathbb{R}$ of the form:

$$
\mathcal{L}(q, \dot{q})=-(1 / 2)\langle\dot{q}, \dot{q}\rangle+\mathcal{V}(q),
$$

where the potential energy $\mathcal{V}$ admits directional derivatives. The directional derivative of $\mathcal{V}$ at $q \in X$ in direction $q^{\prime} \in X$ is denoted $\mathrm{D} \mathcal{V}(q) q^{\prime}$. The EulerLagrange equations for a function $t \mapsto q(t) \in X$ are equivalent to the system of first order equations:

$$
\begin{aligned}
\dot{q} & =-p \\
\forall p^{\prime} \in X \quad\left\langle\dot{p}, p^{\prime}\right\rangle & =\mathrm{D} \mathcal{V}(q) p^{\prime} .
\end{aligned}
$$


These are the equations associated with the Hamiltonian:

$$
\mathcal{H}(q, p)=(1 / 2)\langle p, p\rangle+\mathcal{V}(q) .
$$

In particular, given any solution, the function $t \mapsto \mathcal{H}(q(t), p(t))$ is constant.

Suppose that we have an action of a Lie group $G$ on $X$, i.e. a map $\mathcal{G}$ : $G \times X \rightarrow X:(\beta, q) \mapsto \mathcal{G}_{\beta}(q)$, such that $\mathcal{L}$ is invariant under $\mathcal{G}$. That is, for all $\beta \in G$ and all $q, q^{\prime} \in X$ :

$$
\mathcal{L}\left(\mathcal{G}_{\beta}(q), \mathrm{DG}_{\beta}(q) q^{\prime}\right)=\mathcal{L}\left(q, q^{\prime}\right) .
$$

Let $\mathfrak{g}$ be the Lie algebra of $G$. We introduce the infinitesimal generators $\Gamma$ : $X \rightarrow \mathfrak{g}^{\star}$ defined by:

$$
\Gamma(q) \beta^{\prime}=\left.\partial_{\beta^{\prime}} \mathcal{G}_{\beta}(q)\right|_{\beta=1} .
$$

Notice that in this section we have a multiplicative, possibly non-commutative, group whereas for the Maxwell-Klein-Gordon we had an additive commutative group. We have the well-known Noether's theorem (see e.g. [20]:

Proposition 3.4 Suppose $t \mapsto(p(t), q(t))$ solves (81). Then for each $\beta^{\prime} \in \mathfrak{g}$ the function $t \mapsto\left\langle p(t), \Gamma(q(t)) \beta^{\prime}\right\rangle$ is constant.

Suppose we have a finite dimensional subspace $X_{\sigma} \subset X$. A function $t \mapsto$ $q(t) \in X_{\sigma}$ is a critical point of the action associated with the Lagrangian $\mathcal{L}$, restricted to functions $\mathbb{R} \rightarrow X_{\sigma}$, if and only if the following equations are satisfied:

$$
\begin{aligned}
\dot{q} & =-p, \\
\forall p^{\prime} \in X_{\sigma} \quad\left\langle\dot{p}, p^{\prime}\right\rangle & =\mathrm{DV}(q) p^{\prime} .
\end{aligned}
$$

Suppose we have a subgroup $G_{\sigma} \subset G$ with Lie algebra $\mathfrak{g}_{\sigma}$. If $G_{\sigma}$ leaves $X_{\sigma}$ invariant then the functions $t \mapsto\left\langle p(t), \Gamma(q(t)) \beta^{\prime}\right\rangle$ are constant for each $\beta^{\prime} \in \mathfrak{g}_{\sigma}$. However if $G_{\beta}$ does not map $X_{\sigma}$ to $X_{\sigma}$ this is no longer true, as we observed numerically for the MKG equation. Our situation is rather that for each $q \in X_{\sigma}$ the map $\mathfrak{g}_{\sigma} \rightarrow X_{\sigma}^{\star}: \beta^{\prime} \mapsto\left\langle\cdot, \Gamma(q) \beta^{\prime}\right\rangle$ is onto.

We suppose that the kinetic and potential terms of the Lagrangian are separately invariant under $G$. That is, for each $\beta \in G$ each $q, q^{\prime} \in X$ :

$$
\begin{aligned}
\left\langle\mathrm{DG}_{\beta}(q) q^{\prime}, \mathrm{D} \mathcal{G}_{\beta}(q) q^{\prime}\right\rangle & =\left\langle q^{\prime}, q^{\prime}\right\rangle, \\
\mathcal{V}\left(\mathcal{G}_{\beta}(q)\right) & =\mathcal{V}(q) .
\end{aligned}
$$

The infinitesimal versions of these equations are, for each $\beta^{\prime} \in \mathfrak{g}$ and each $q, q^{\prime} \in X$ :

$$
\begin{aligned}
\left\langle\mathrm{D} \Gamma(q) q^{\prime} \beta^{\prime}, q^{\prime}\right\rangle & =0 \\
\mathrm{D} \mathcal{V}(q) \Gamma(q) \beta^{\prime} & =0 .
\end{aligned}
$$

In these circumstances we consider the differential equation, for a map $t \mapsto$ $(p(t), q(t)) \in X_{\sigma} \times X_{\sigma}$ and a Lagrange multiplier $\beta(t) \in \mathfrak{g}:$

$$
\begin{aligned}
& \begin{aligned}
\dot{q} & =-p \\
\forall p^{\prime} \in X_{\sigma} \quad\left\langle\dot{p}, p^{\prime}\right\rangle+\left\langle p^{\prime}, \Gamma(q) \beta\right\rangle & =\mathrm{D} \mathcal{V}(q) p^{\prime},
\end{aligned} \\
& \forall \beta^{\prime} \in \mathfrak{g}_{\sigma} \quad\left\langle\dot{p}, \Gamma(q) \beta^{\prime}\right\rangle=0 .
\end{aligned}
$$


Proposition 3.5 For each solution of the system (90) and each $\beta^{\prime} \in \mathfrak{g}_{\sigma}$, the function $t \mapsto\left\langle p(t), \Gamma(q(t)) \beta^{\prime}\right\rangle$ is constant. If the constraint is 0 initially, the Hamiltonian is also conserved.

- Proof: We have:

$$
\left\langle p, \Gamma(q) \beta^{\prime}\right\rangle^{\cdot}=\left\langle\dot{p}, \Gamma(q) \beta^{\prime}\right\rangle+\left\langle p, \Gamma(q)^{\cdot} \beta^{\prime}\right\rangle .
$$

The first term on the right hand side is 0 by construction. For the second we remark that:

$$
\begin{aligned}
\left\langle p, \Gamma(q)^{\cdot} \beta^{\prime}\right\rangle & =\left\langle p, \mathrm{D} \Gamma(q) \dot{q} \beta^{\prime}\right\rangle, \\
& =-\left\langle p, \mathrm{D} \Gamma(q) p \beta^{\prime}\right\rangle, \\
& =0 .
\end{aligned}
$$

This proves the first part of the proposition. For the second we remark that:

$$
\begin{aligned}
\mathcal{H}(q, p)^{\circ} & =\langle\dot{p}, p\rangle+\mathrm{D} \mathcal{V}(q) \dot{q} \\
& =\mathrm{D} \mathcal{V}(q) p-\langle p, \Gamma(q) \beta\rangle-\mathrm{D} \mathcal{V}(q) p \\
& =0
\end{aligned}
$$

This concludes the proof.

We now turn to time-discretizations. The time-step is denoted $\tau$. Consider sequences of vectors $\left(p_{n+1 / 2}, q_{n}\right) \in X_{\sigma} \times X_{\sigma}$ such that:

$$
\begin{aligned}
(1 / \tau)\left(q_{n}-q_{n-1}\right) & =-p_{n-1 / 2}, \\
(1 / \tau)\left(p_{n+1 / 2}-p_{n-1 / 2}\right) & =\dot{p}_{n},
\end{aligned}
$$

where $\dot{p}_{n} \in X_{\sigma}$ is the solution of the system, with Lagrange multiplier $\beta_{n} \in \mathfrak{g}_{\sigma}$ :

$$
\begin{array}{lll}
\forall p^{\prime} \in X_{\sigma} \quad\left\langle\dot{p}_{n}, p^{\prime}\right\rangle+\left\langle p^{\prime}, \Gamma\left(q_{n}\right) \beta_{n}\right\rangle & =\mathrm{D} \mathcal{V}\left(q_{n}\right) p^{\prime}, \\
\forall \beta^{\prime} \in \mathfrak{g}_{\sigma} & \left\langle\dot{p}_{n}, \Gamma\left(q_{n}\right) \beta^{\prime}\right\rangle & =
\end{array}
$$

We remark that:

$$
\left\langle p_{n+1 / 2}, \Gamma\left(q_{n}\right) \beta^{\prime}\right\rangle=\left\langle\frac{p_{n+1 / 2}+p_{n-1 / 2}}{2}, \Gamma\left(q_{n}\right) \beta^{\prime}\right\rangle .
$$

We have:

Proposition 3.6 Suppose that for each $\beta^{\prime}$ the map $q \mapsto \Gamma(q) \beta^{\prime}$ is a polynomial of degree at most 2 . Then for each $\beta^{\prime} \in \mathfrak{g}$ the quantities $\left\langle p_{n+1 / 2}, \Gamma\left(q_{n}\right) \beta^{\prime}\right\rangle$ are conserved.

- Proof: Indeed we have:

$$
\begin{aligned}
& \left\langle p_{n+1 / 2}, \Gamma\left(q_{n}\right) \beta^{\prime}\right\rangle \\
= & \left\langle p_{n-1 / 2}, \Gamma\left(q_{n}\right) \beta^{\prime}\right\rangle \\
= & \left\langle p_{n-1 / 2}, \Gamma\left(q_{n-1}\right) \beta^{\prime}+\mathrm{D} \Gamma\left((1 / 2)\left(q_{n-1}+q_{n}\right)\right)\left(q_{n}-q_{n-1}\right) \beta^{\prime}\right\rangle .
\end{aligned}
$$

Moreover:

$$
\begin{aligned}
& \left\langle p_{n-1 / 2}, \mathrm{D} \Gamma\left((1 / 2)\left(q_{n-1}+q_{n}\right)\right)\left(q_{n}-q_{n-1}\right) p_{n-1 / 2}\right\rangle \\
= & \left\langle p_{n-1 / 2}, \mathrm{D} \Gamma\left((1 / 2)\left(q_{n-1}+q_{n}\right)\right) \tau p_{n-1 / 2} \beta^{\prime}\right\rangle, \\
= & 0 .
\end{aligned}
$$

This completes the proof. 


\section{Discretization in Lorenz gauge}

\subsection{Mass-lumping the Klein-Gordon equation}

We first make some comments on the discretization of the Klein-Gordon part of the equation. If we look at the action for the MKG equation in general gauge and isolate the part pertaining to the scalar field $\phi$, we are lead to study:

$$
\mathcal{S}(\phi)=(1 / 2) \int-|\dot{\phi}+\alpha \phi|^{2}+\left|\mathrm{D}_{A} \phi\right|^{2} .
$$

We treat, in this section, $\alpha$ and $A$ as given functions $\mathbb{R} \rightarrow Y_{\sigma}^{0}$ and $\mathbb{R} \rightarrow Y_{\sigma}^{1}$. If we look for critical points of the restriction of $\mathcal{S}$ to functions $\phi: \mathbb{R} \rightarrow Z_{\sigma}^{0}$, we run into difficulties in defining the momentum conjugate to $\phi$. Indeed suppose we let $\Pi_{\sigma}$ denote the projection onto $Z_{\sigma}^{0}$ defined by $\langle\cdot, \cdot\rangle$ (the $\mathrm{L}^{2}$ projection). The momentum would be:

$$
\psi=-\dot{\phi}-\Pi_{\sigma}(\alpha \phi)
$$

since this ensures that for all $\psi^{\prime} \in Z_{\sigma}^{0}$ :

$$
\left\langle\psi, \psi^{\prime}\right\rangle=-\left\langle\dot{\phi}+\alpha \phi, \psi^{\prime}\right\rangle
$$

Stationarity of the action is written, for all $\phi^{\prime}: \mathbb{R} \rightarrow Z_{\sigma}^{0}$ :

$$
\int\left\langle\dot{\phi}+\alpha \phi, \dot{\phi}^{\prime}+\alpha \phi^{\prime}\right\rangle=\int\left\langle\mathrm{D}_{A} \phi, \mathrm{D}_{A} \phi^{\prime}\right\rangle .
$$

Since $\alpha \phi^{\prime}$ is not in $Z_{\sigma}^{0}$ there is, as far as I can see, no simple way of writing this as a differential equation on $\psi$.

We resolve this problem by a procedure known as mass-lumping. Let $\Pi_{\sigma}$ denote instead the nodal interpolator on $Z_{\sigma}^{0}$. Define the mass-lumped duality by:

$$
\langle\cdot, \cdot\rangle_{\sigma}:\left(\phi, \phi^{\prime}\right) \mapsto \int \Pi_{\sigma}\left(\phi \phi^{\prime}\right)
$$

This bilinear form is positive definite on $Z_{\sigma}^{0}$. Moreover we have:

$$
\left\langle\phi, \phi^{\prime}\right\rangle_{\sigma}=\left\langle\Pi_{\sigma} \phi, \phi^{\prime}\right\rangle_{\sigma}
$$

In particular $\Pi_{\sigma}$ is the projection onto $Z_{\sigma}^{0}$ defined by $\langle\cdot, \cdot\rangle_{\sigma}$.

Consider now the modified action:

$$
\mathcal{S}_{\sigma}(\phi)=(1 / 2) \int-\langle\dot{\phi}+\alpha \phi, \dot{\phi}+\alpha \phi\rangle_{\sigma}+\left\langle\mathrm{D}_{A} \phi, \mathrm{D}_{A} \phi\right\rangle .
$$

We restrict it to functions $\phi: \mathbb{R} \rightarrow Z_{\sigma}^{0}$. We let $\psi$ denote the momentum conjugate to $\phi$ :

$$
\psi=-\dot{\phi}-\Pi_{\sigma}(\alpha \phi),
$$

Now the stationarity of the action is written:

$$
\int\left\langle\dot{\phi}+\alpha \phi, \dot{\phi}^{\prime}+\alpha \phi^{\prime}\right\rangle_{\sigma}=\int\left\langle\mathrm{D}_{A} \phi, \mathrm{D}_{A} \phi^{\prime}\right\rangle
$$


It is equivalent to the differential equation:

$$
\left\langle\dot{\psi}+\Pi_{\sigma}(\alpha \psi), \phi^{\prime}\right\rangle_{\sigma}=\left\langle\mathrm{D}_{A} \phi, \mathrm{D}_{A} \phi^{\prime}\right\rangle .
$$

A crucial property of the multiplication by $\alpha$ is that it is antisymmetric with respect to the $\mathrm{L}^{2}$ scalar product $\langle\cdot, \cdot\rangle$ :

$$
\left\langle\phi, \alpha \phi^{\prime}\right\rangle=-\left\langle\alpha \phi, \phi^{\prime}\right\rangle .
$$

Therefore differential equations of the form:

$$
\dot{\phi}+\alpha \phi=0,
$$

preserve the $\mathrm{L}^{2}$ norm of $\phi$. We would like to keep this property after discretization.

We luckily have:

$$
\left\langle\phi, \Pi_{\sigma}\left(\alpha \phi^{\prime}\right)\right\rangle_{\sigma}=-\left\langle\Pi_{\sigma}(\alpha \phi), \phi^{\prime}\right\rangle_{\sigma},
$$

therefore, for equations of the form:

$$
\dot{\phi}+\Pi_{\sigma}(\alpha \phi)=0,
$$

the mass-lumped norm associated with $\langle\cdot, \cdot\rangle_{\sigma}$ - which will be denoted $|\cdot|_{\sigma}$ - is preserved. This property can be mimicked in the time-discretized case by the implicit midpoint rule. The cost of the implicit mid-point rule is small since the mass matrix, being lumped, is diagonal.

\subsection{Space-discretization of the full system}

We are interested in the system of equations:

$$
\begin{aligned}
\dot{A} & =-E+\mathrm{d} \alpha \\
\dot{\phi}+\alpha \phi & =-\psi \\
\dot{E} & =\mathrm{d}^{\star} \mathrm{d} A+\Im\left(\mathrm{D}_{A} \phi \bar{\phi}\right), \\
\dot{\psi}+\alpha \psi & =\mathrm{D}_{A}^{\star} \mathrm{D}_{A} \phi
\end{aligned}
$$

For the time-being we suppose that $\alpha$ is some given function. We consider first semi-discretization of the form $\alpha(t) \in Y_{\sigma}^{0}, A(t), E(t) \in Y_{\sigma}^{1}$ and $\phi(t), \psi(t) \in Z_{\sigma}^{0}$. We impose:

$$
\begin{aligned}
\dot{A} & =-E+\mathrm{d} \alpha, \\
\dot{\phi}+\Pi_{\sigma}(\alpha \phi) & =-\psi .
\end{aligned}
$$

We first consider variational discretizations without constraints:

$$
\begin{aligned}
\left\langle\dot{E}, E^{\prime}\right\rangle & =f_{t}\left(E^{\prime}\right) \\
\left\langle\psi^{\circ}, \psi^{\prime}\right\rangle_{\sigma} & =g_{t}\left(\psi^{\prime}\right) .
\end{aligned}
$$

The right hand sides were defined in (48) and (49). We impose:

$$
\dot{\psi}+\Pi_{\sigma}(\alpha \psi)=\psi^{\circ} .
$$


From the study of the case $\alpha=0$ we know that we should not expect constraint preservation for this semi-discretization. Curiously we don't even have energy conservation. The mass-lumped Hamiltonian is defined by:

$$
\mathcal{H}_{\sigma}(A, \phi, E, \psi)=(1 / 2)\left(|E|^{2}+|\mathrm{d} A|^{2}+|\psi|_{\sigma}^{2}+\left|\mathrm{D}_{A} \phi\right|^{2}\right) .
$$

Proposition 4.1 For solutions of (126 - 130) we have:

$$
\dot{\mathcal{H}}_{\sigma}=\langle\dot{E}, \mathrm{~d} \alpha\rangle-\left\langle\psi^{\circ}, \alpha \phi\right\rangle_{\sigma} .
$$

- Proof: Inserting $E^{\prime}=\dot{A}$ in (128)and $\psi^{\prime}=\dot{\phi}$ in (129), and adding we get:

$$
\begin{aligned}
& (1 / 2)\langle\mathrm{d} A, \mathrm{~d} A\rangle^{\cdot}+(1 / 2)\left\langle\mathrm{D}_{A} \phi, \mathrm{D}_{A} \phi\right\rangle^{\circ} \\
= & \langle\dot{E}, \dot{A}\rangle+\left\langle\psi^{\circ}, \dot{\phi}\right\rangle_{\sigma}, \\
= & \langle\dot{E},-E+\mathrm{d} \alpha\rangle+\left\langle\psi^{\circ},-\Pi_{\sigma}(\alpha \phi)-\psi\right\rangle_{\sigma}, \\
= & -(1 / 2)\langle E, E\rangle^{\cdot}-(1 / 2)\langle\psi, \psi\rangle_{\sigma}+\langle\dot{E}, \mathrm{~d} \alpha\rangle-\left\langle\psi^{\circ}, \alpha \phi\right\rangle_{\sigma} .
\end{aligned}
$$

The equality follows.

We now introduce constrained formulations. We keep $(126,127)$ but instead of $(128,129)$ we solve the system in $\left(\dot{E}, \psi^{\circ}\right)$ :

$$
\begin{array}{ll}
\left\langle\dot{E}, E^{\prime}\right\rangle+\left\langle\psi^{\circ}, \psi^{\prime}\right\rangle_{\sigma}+\left\langle E^{\prime}, \mathrm{d} \beta\right\rangle-\left\langle\psi^{\prime}, \phi \beta\right\rangle_{\sigma} & =f_{t}\left(E^{\prime}\right)+g_{t}\left(\psi^{\prime}\right), \\
\left\langle\dot{E}, \mathrm{~d} \beta^{\prime}\right\rangle-\left\langle\psi^{\circ}, \phi \beta^{\prime}\right\rangle_{\sigma} & =0,
\end{array}
$$

with $f_{t}$ and $g_{t}$ defined, as before, by equations $(48,49)$. We impose $(130)$ as before.

We have:

Proposition 4.2 Equations (126, 127, 137, 130) preserve the constraint:

$$
\left\langle E, \mathrm{~d} \beta^{\prime}\right\rangle-\left\langle\psi, \phi \beta^{\prime}\right\rangle_{\sigma}=0 .
$$

- Proof: We compute:

$$
\begin{aligned}
& \left(\left\langle E, \mathrm{~d} \beta^{\prime}\right\rangle-\left\langle\psi, \phi \beta^{\prime}\right\rangle_{\sigma}\right)^{\cdot} \\
= & \left\langle\dot{E}, \mathrm{~d} \beta^{\prime}\right\rangle-\left\langle\dot{\psi}, \phi \beta^{\prime}\right\rangle_{\sigma}-\left\langle\psi, \dot{\phi} \beta^{\prime}\right\rangle_{\sigma}, \\
= & \left\langle\dot{E}, \mathrm{~d} \beta^{\prime}\right\rangle-\left\langle\psi^{\circ}, \phi \beta^{\prime}\right\rangle_{\sigma}+\left\langle\alpha \psi, \phi \beta^{\prime}\right\rangle_{\sigma}+\left\langle\psi, \psi \beta^{\prime}\right\rangle_{\sigma}+\left\langle\psi, \alpha \phi \beta^{\prime}\right\rangle_{\sigma}, \\
= & 0 .
\end{aligned}
$$

This completes the proof.

Concerning energy we have:

Proposition 4.3 Suppose equations (126, 127, 137, 130) are satisfied as well as the constraint (138). The mass-lumped Hamiltonian has the property:

$$
\dot{\mathcal{H}}_{\sigma}=\langle\mathrm{d} \alpha, \mathrm{d} \beta\rangle+\langle\alpha \phi, \phi \beta\rangle_{\sigma} .
$$

- Proof: We insert $E^{\prime}=\dot{A}$ and $\psi^{\prime}=\dot{\phi}$ in (137), obtaining:

$$
\begin{aligned}
& \langle\dot{E}, \dot{A}\rangle+\langle\dot{\psi}+\alpha \psi, \dot{\phi}\rangle_{\sigma}+\langle\dot{A}, \mathrm{~d} \beta\rangle-\langle\dot{\phi}, \phi \beta\rangle_{\sigma} \\
= & \langle\mathrm{d} A, \mathrm{~d} \dot{A}\rangle+\left\langle\mathrm{D}_{A} \phi, \phi \dot{A}\right\rangle+\left\langle\mathrm{D}_{A} \phi, \mathrm{D}_{A} \dot{\phi}\right\rangle .
\end{aligned}
$$


By $(126,127)$ the right-hand side is equal to:

$$
\langle\dot{E},-E+\mathrm{d} \alpha\rangle+\langle\dot{\psi}+\alpha \psi,-\psi-\alpha \phi\rangle_{\sigma}+\langle-E+\mathrm{d} \alpha, \mathrm{d} \beta\rangle+\langle\psi+\alpha \phi, \phi \beta\rangle_{\sigma} .
$$

Using the constraint we get:

$$
\begin{aligned}
& -1 / 2\langle E, E\rangle^{\cdot}-1 / 2\langle\psi, \psi\rangle_{\sigma}+\langle\mathrm{d} \alpha, \mathrm{d} \beta\rangle+\langle\alpha \phi, \phi \beta\rangle_{\sigma} \\
= & 1 / 2\langle\mathrm{~d} A, \mathrm{~d} A\rangle^{\cdot}+1 / 2\left\langle\mathrm{D}_{A} \phi, \mathrm{D}_{A} \phi\right\rangle^{\prime} .
\end{aligned}
$$

This completes the proof.

\subsection{Time-discretization of the full system}

We perform the following time-steps. In parallel to (126) we impose:

$$
(1 / \tau)\left(A_{n}-A_{n-1}\right)=-E_{n-1 / 2}+\mathrm{d} \alpha_{n-1 / 2},
$$

next, in analogy with (127) we enforce:

$$
(1 / \tau)\left(\phi_{n}-\phi_{n-1}\right)+\Pi_{\sigma}\left(\alpha_{n-1 / 2}(1 / 2)\left(\phi_{n}+\phi_{n-1}\right)\right)=-\psi_{n-1 / 2} .
$$

Then (for an unconstrained simulation), in analogy with $(128,129)$ we determine $\dot{E}_{n}$ and $\psi_{n}^{\circ}$ by:

$$
\left\langle\dot{E}_{n}, E^{\prime}\right\rangle=f_{n}\left(E^{\prime}\right)
$$

and:

$$
\left\langle\psi_{n}^{\circ}, \psi^{\prime}\right\rangle_{\sigma}=g_{n}\left(\psi^{\prime}\right)
$$

We follow up with:

$$
(1 / \tau)\left(E_{n+1 / 2}-E_{n-1 / 2}\right)=\dot{E}_{n},
$$

and:

$$
(1 / \tau)\left(\psi_{n+1 / 2}-\psi_{n-1 / 2}\right)+\Pi_{\sigma}\left(\alpha_{n}(1 / 2)\left(\psi_{n+1 / 2}+\psi_{n-1 / 2}\right)\right)=\psi_{n}^{\circ},
$$

where:

$$
\alpha_{n}=(1 / 2)\left(\alpha_{n+1 / 2}+\alpha_{n-1 / 2}\right) .
$$

At this point we suppose that $\alpha_{n+1 / 2}$ has been determined by the choice of gauge. We post-pone the discussion of gauging to the next paragraph.

For the constrained scheme, in analogy with (137), we determine instead $\dot{E}_{n}$ and $\psi_{n}^{\circ}$ by the following system with Lagrange multiplier $\beta_{n}$ :

$$
\begin{array}{ll}
\left\langle\dot{E}_{n}, E^{\prime}\right\rangle+\left\langle\psi_{n}^{\circ}, \psi^{\prime}\right\rangle_{\sigma}+\left\langle E^{\prime}, \mathrm{d} \beta_{n}\right\rangle-\left\langle\psi^{\prime}, \phi_{n} \beta_{n}\right\rangle_{\sigma} & =f_{n}\left(E^{\prime}\right)+g_{n}\left(\psi^{\prime}\right), \\
\left\langle\dot{E}_{n}, \mathrm{~d} \beta^{\prime}\right\rangle-\left\langle\psi_{n}^{\circ}, \phi_{n} \beta^{\prime}\right\rangle_{\sigma} & =0,
\end{array}
$$

\subsection{Gauging}

The Lorenz gauge consists in determining $\alpha$ by the condition:

$$
\dot{\alpha}=-\mathrm{d}^{\star} A .
$$

In the discrete setting we impose:

$$
\left\langle(1 / \tau)\left(\alpha_{n+1 / 2}-\alpha_{n-1 / 2}\right), \alpha^{\prime}\right\rangle=-\left\langle A_{n}, \mathrm{~d} \alpha^{\prime}\right\rangle .
$$




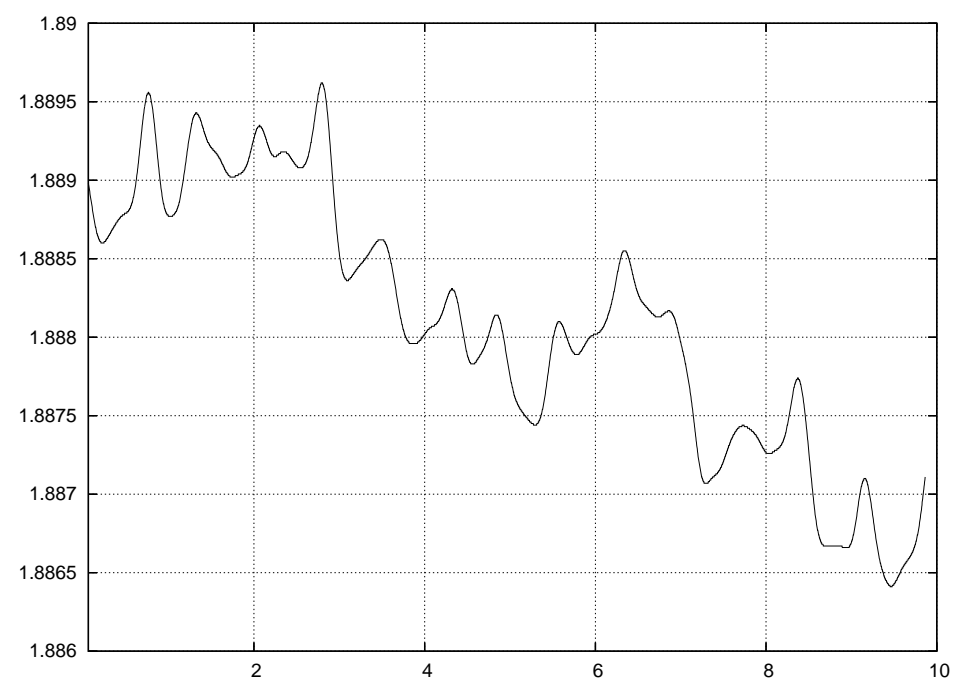

Figure 5: Energy; unconstrained, Lorenz gauge.

We first perform some experiments with the unconstrained scheme. In Figure 5 we plot the energy of the solution. The discrete energy is defined by masslumping the one we used for the temporal gauge:

$$
2 \mathcal{H}_{n}=\left|\frac{E_{n+1 / 2}+E_{n-1 / 2}}{2}\right|^{2}+\left|\mathrm{d} A_{n}\right|^{2}+\left|\frac{\psi_{n+1 / 2}+\psi_{n-1 / 2}}{2}\right|_{\sigma}^{2}+\left|\mathrm{D}_{A_{n}} \phi_{n}\right|^{2} .
$$

The energy appears to be a bit erratic but varies about $0.2 \%$ over a relatively long time interval. We think this is acceptable. Next, in Figure 6 we plot the $\mathrm{L}^{2}$ norms squared of the constraint field $\gamma_{n}$ and of the divergence of the electric field $\delta_{n}$. They are defined by:

$$
\left\langle\gamma_{n}, \beta^{\prime}\right\rangle=\left\langle\frac{E_{n+1 / 2}+E_{n-1 / 2}}{2}, \mathrm{~d} \beta^{\prime}\right\rangle-\left\langle\frac{\psi_{n+1 / 2}+\psi_{n-1 / 2}}{2}, \phi_{n} \beta^{\prime}\right\rangle_{\sigma},
$$

and:

$$
\left\langle\delta_{n}, \beta^{\prime}\right\rangle=\left\langle\frac{E_{n+1 / 2}+E_{n-1 / 2}}{2}, \mathrm{~d} \beta^{\prime}\right\rangle .
$$

As was the case for the temporal gauge, the constraint is not satisfied.

Then we turn our attention to the constrained scheme. In Figure 7 we plot the energy of the solution. Curiously the plot of the energy is nearly identical to the one obtained without constraints. In Figure 8 we plot the $\mathrm{L}^{2}$ norms of the constraint field and the divergence of the electric field. The constraint is now satisfied. 


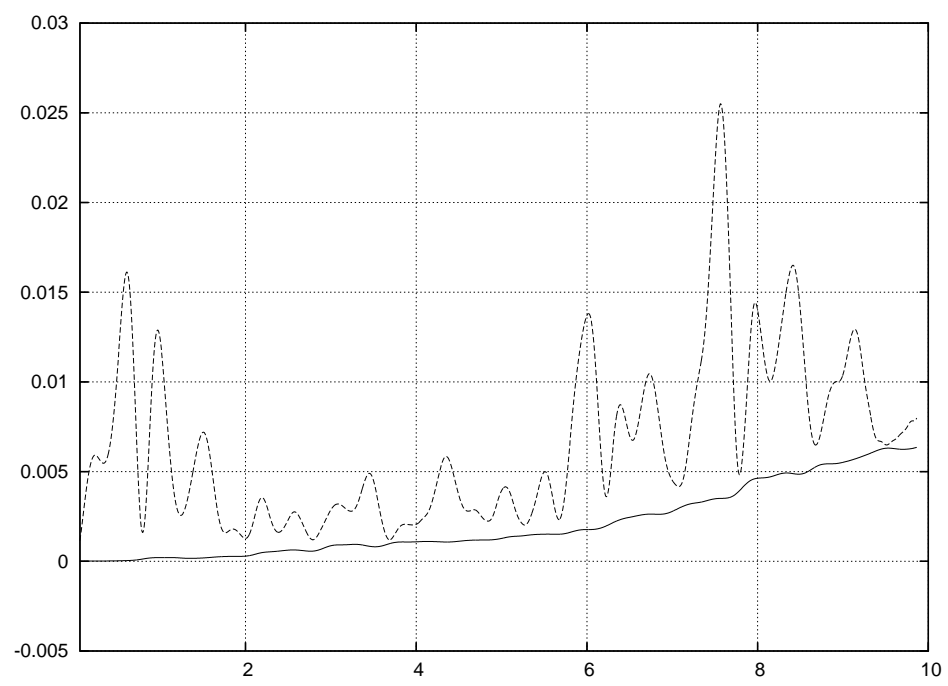

Figure 6: Constraint (plain) and divergence (dashed); unconstrained, Lorenz gauge.

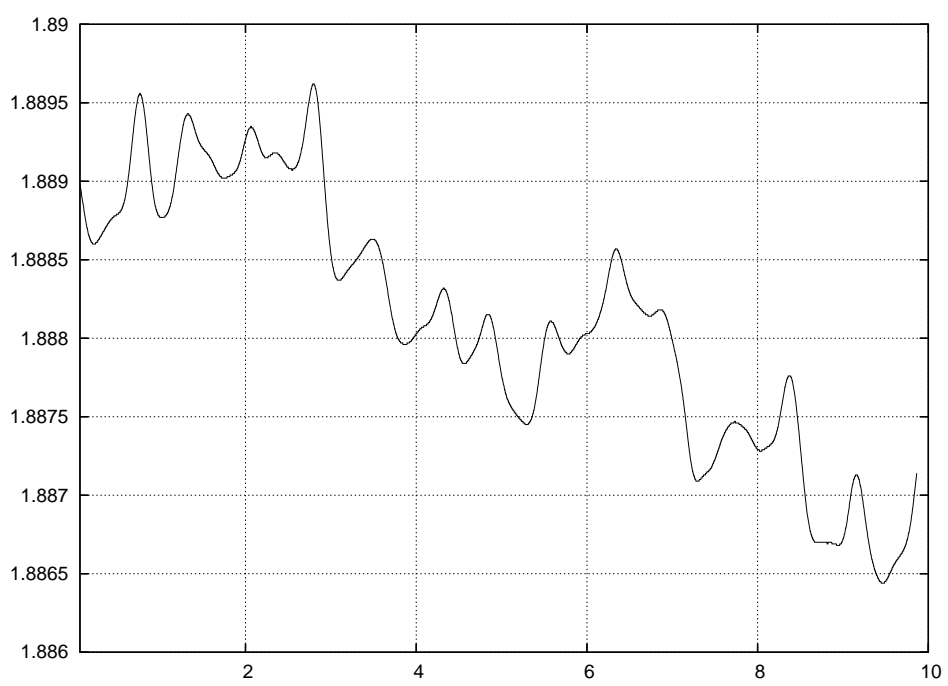

Figure 7: Energy; constrained, Lorenz gauge. 


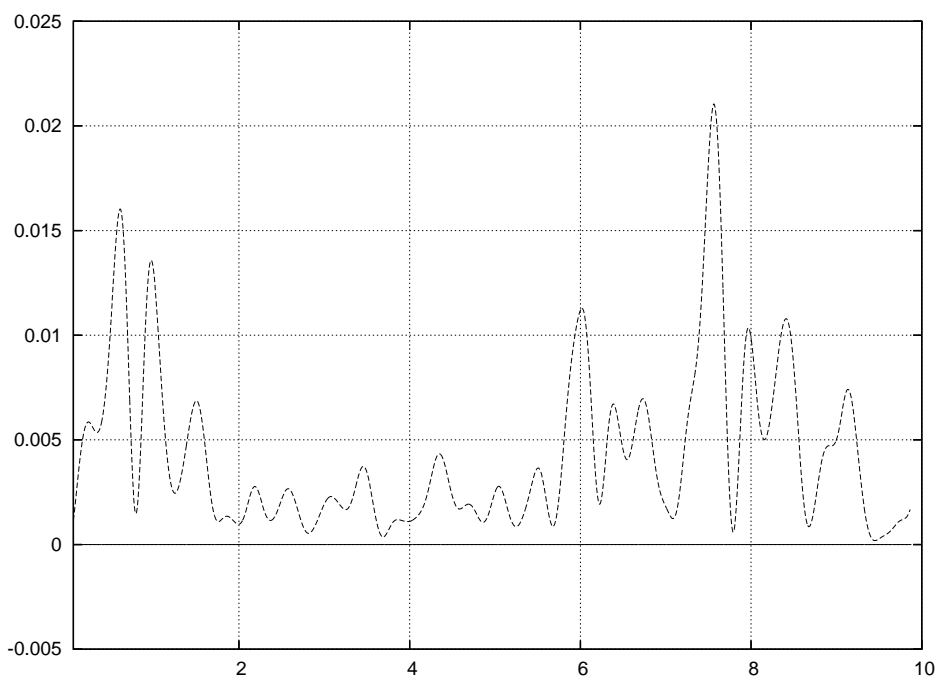

Figure 8: Constraint (plain) and divergence (dashed); constrained, Lorenz gauge.

\section{Concluding remarks}

One tempting correction is to modify the gauging of $\alpha$ in such a way that $\langle\mathrm{d} \alpha, \mathrm{d} \beta\rangle+\langle\alpha \phi, \phi \beta\rangle_{\sigma}=0$. This could lead to exact energy conservation without interfering with the constraint. We have not reached conclusive results on this line of thought. We notice that this condition is in some sense ill-conditioned since the Lagrange multiplier $\beta$ is a small and wild field.

It also appears that the method of constraints introduced in this paper applies to equations with a different structure. In particular we have checked this on the Maxwell-Schrödinger equation.

\section{Acknowledgments}

My interest in the Maxwell-Klein-Gordon equation was spurred by a conversation with Sigmund Selberg at the Mittag-Leffler Institute during the Fall 2005 program "Wave motion". I completed the paper while a visitor at the Isaac Newton Institute during the Spring 2007 program "Highly oscillatory problems".

I thank Jörg Frauendiener for his comments.

This work, conducted as part of the award "Numerical analysis and simulations of geometric wave equations" made under the European Heads of Research Councils and European Science Foundation EURYI (European Young Investigator) Awards scheme, was supported by funds from the Participating Organizations of EURYI and the EC Sixth Framework Program. 


\section{References}

[1] D.N. ARnold: Differential complexes and numerical stability; Plenary address delivered at ICM 2002, International Congress of Mathematicians, Beijing 2002.

[2] D.N. Arnold, R.S. FAlK, R. Winther: Finite element exterior calculus, homological techniques, and applications; Acta Numerica, Vol. 15, p. $1-155,2006$.

[3] D. Boffi, A. Buffa, L. Gastaldi: Convergence analysis for hyperbolic evolution problems in mixed form; IMATI Technical Report, No. 17-PV, 2005.

[4] D. Boffi, P. Fernandes, L. Gastaldi, I. Perugia: Computational models of electromagnetic resonators: analysis of edge element approximation; SIAM J. Numer. Anal., Vol. 36, No. 4, p. 1264 - 1290, 1999.

[5] D. Boffi, L. Gastaldi: Analysis of finite element approximation of evolution problems in mixed form; SIAM J. Numer. Anal., Vol. 42, No. 4, p. $1502-1526,2004$.

[6] A. Bossavit: Mixed finite elements and the complex of Whitney forms; The mathematics of finite elements and applications, VI (Uxbridge, 1987), p. 137 - 144, Academic Press, London, 1988.

[7] F. BREzzI: On the existence, uniqueness and approximation of saddlepoint problems arising from Lagrangian multipliers; RAIRO Anal. Numér., Vol. 8, No. R-2, p. 129 - 151, 1974.

[8] F. Brezzi, M. Fortin: Mixed and hybrid finite element methods; Springer-Verlag, 1991.

[9] S.H. Christiansen, R. Winther: On constraint preservation in numerical simulations of Yang-Mills equations; SIAM J. Sci. Comp., Vol. 28, No. 1, p. $75-101,2006$.

[10] P. Ciarlet JR., J. Zou: Fully discrete finite element approaches for time-dependent Maxwell's equations; Numer. Math., Vol. 82, p. 193 - 219, 1999.

[11] D. Eardley, V. Moncrief: The global existence of Yang-Mills-Higgs fields in $\mathrm{M}^{3+1}$; Comm. Math. Phys., Vol. 83, p. $171-212,1982$.

[12] V. Girault, P.-A. Raviart: Finite element methods for Navier-Stokes equations; Springer-Verlag, Berlin, 1986.

[13] E. Hairer, C. Lubich, G. Wanner: Geometric numerical integration: structure preserving algorithms for ordinary differential equations; Springer Series in Computational Mathematics, No. 31, Springer, 2002.

[14] R. Hiptmair: Finite elements in computational electromagnetism; Acta Numerica, p. 237 - 339, Cambridge University Press, 2002. 
[15] P. JoLY: Variational methods for time-dependent wave propagation problems; Topics in computational wave propagation, p. 201-264, Lect. Notes Comput. Sci. Eng., 31, Springer, Berlin, 2003..

[16] F. KIKUCHI: On a discrete compactness property for the Nédélec finite elements; J. Fac. Sci. Univ. Tokyo, Sect. 1A Math., Vol. 36, p. 479 - 490, 1989.

[17] S. Lang: Differential and Riemannian manifolds, Third edition; SpringerVerlag, New York, 1995.

[18] L. LehneR: Numerical relativity: a review; Class. Quantum Grav., Vol. 18 , p. $25-86,2001$

[19] B. Leimkuhler And S. Reich: Simulating Hamiltonian dynamics; Cambridge Monographs on Applied and Computational Mathematics, Vol. 14, Cambridge University Press, 2004.

[20] J. E. MARsden, M. West: Discrete mechanics and variational integrators; Acta Numerica, p. 357 - 514, Cambridge University Press, 2001.

[21] P. Monk: Finite element methods for Maxwell's equations; Oxford University Press, 2003.

[22] J.-C. NÉDÉLEC: Mixed finite elements in $\mathbb{R}^{3}$; Numer. Math., Vol. 35, p. $315-341,1980$.

[23] J.E. Roberts, J.-M. Thomas: Mixed and hybrid methods; p. 523-640, in P.G. Ciarlet, J.-L. Lions (eds.), "Handbook of numerical analysis, Vol. II, Finite element methods (Part 1)", North-Holland, 1991.

[24] R. SoRkin: The electromagnetic field on a simplicial net; J. Math. Phys., Vol. 16, No. 12, p. 2432 - 2440, 1975.

[25] R. M. WALD: General relativity; University of Chicago Press, 1984.

[26] H. Whitney: Geometric integration theory; Princeton University Press, 1957. 